\title{
El significado de la vida y de la muerte para mujeres con cáncer de mama
}

\section{The Meaning of Life and the Meaning of Death for Women with Breast Cancer 0 significado da vida e da morte para mulheres com cáncer de mama}

\author{
Ximena Palacios-Espinosa*, Ana Milena Lizarazo*, \\ Karen Stephanie Moreno*, Julio David Ospino* \\ Universidad del Rosario, Bogotá, Colombia
}

Doi: dx.doi.org/10.12804/apl33.03.2015.07

\section{Resumen}

Tener cáncer promueve reflexiones y cambios en el significado de la vida y de la muerte. Objetivo: Esta investigación cualitativa tuvo como objetivo comprender el significado de la vida y la muerte en un grupo de once mujeres con cáncer de mama. Método: Se realizó una entrevista a cada participante y se clasificó y codificó la información buscando categorías emergentes con base en el enfoque procesual de las representaciones sociales. Resultados: Algunas de las categorías fueron: apoyo, tratamiento, muerte; efectos secundarios; afrontamiento y suicidio; experiencia positiva con el médico y el hospital; reacciones emocionales; conocimiento previo de la quimioterapia; experiencias previas con el cáncer; proceso de diagnóstico y médico como poseedor de conocimiento. Conclusiones: Este estudio permitió aproximarse a la comprensión tanto del cáncer de mama como de los cambios que este genera en el significado de la vida y de la muerte. Así mismo, permitió comprender la importancia de la familia y de la red de apoyo en esta experiencia y el sentimiento de lucha y fortalecimiento del rol social de mujer para afrontar la enfermedad. Palabras clave: cáncer de mama; representaciones sociales; sentido de vida; sentido de muerte.

\section{fbstract}

Having cancer promotes reflections and changes in the meaning of life and death. Objective: This qualitative study aimed to understand the meaning of life and death in a group of 11 women with breast cancer. Method: An interview with each participant was conducted and the information was classified and coded seeking emerging categories based on the procedural approach of Social Representations. Results: Some of the categories were: support, treatment, death; side effects; coping and suicide; positive experience with the doctor and the hospital; emotional reactions; prior knowledge of chemotherapy; previous experiences with cancer; and medical diagnostic process as having knowledge. Conclusions: This study allowed to approach an understanding of

* Ximena Palacios-Espinosa, Ana Milena Lizarazo, Karen Stephanie Moreno y Julio David Ospino, Programa de Psicología, Universidad del Rosario

La correspondencia relacionada con este artículo debe ser enviada a Ximena Palacios-Espinosa. Correo electrónico: ximena.palacios@urosario.edu.co

Declaración: los autores de este artículo declaran que no tienen conflictos de intereses y que conocen y aprueban su contenido.

Cómo citar este artículo: Palacios-Espinosa, X., Lizarazo, A. M., Moreno, K. S. \& Ospino, J. D. (2015). El significado de la vida y de la muerte para mujeres con cáncer de mama. Avances en Psicología Latinoamericana, 33(3), 455-479. doi: dx.doi. org/10.12804/apl33.03.2015.07 
both breast cancer and the changes it generates in the meaning of life and death. Also, it allowed to understand the importance of family and the support network in this experience and feeling of struggle and strengthening the social role of women in coping with the disease.

Keywords: breast cancer; social representations; meaning of life; meaning of death.

\section{Resumo}

Ter cáncer promove reflexões e mudanças no significado da vida e da morte. Objetivo: esta pesquisa qualitativa, teve como objetivo compreender o significado da vida e a morte em um grupo de 11 mulheres com cáncer de mama. Método: realizou-se uma entrevista a cada participante e se classificou e codificou a informação buscando categorias emergentes com base no enfoque processual das Representações Sociais. Resultados: algumas das categorias foram: apoio, tratamento, morte; efeitos secundários; afrontamento e suicídio; experiência positiva com o médico e o hospital; reações emocionais; conhecimento prévio da quimioterapia; experiências prévias com o cáncer; processo de diagnóstico e médico como possuidor de conhecimento. Conclusões: este estudo permitiu a aproximação à compreensão tanto do cáncer de mama quanto das mudanças que este gera no significado da vida e da morte. Igualmente, permitiu compreender a importância da família e da rede de apoio nesta experiência e o sentimento de luta e fortalecimento do rol social de mulheres para afrontar a doença.

Palavras-chave: cáncer de mama; representações sociais; sentido de vida; sentido de morte.

La literatura sobre los aspectos psicológicos del cáncer de mama en mujeres es amplia (Dupont, Bower, Stanton \& Ganz, 2014; Fernandes, Cruz, Moreira, Santos \& Silva, 2014). No igual, aquella que refiere a la comprensión de lo que la vida y la muerte significan para una mujer con este diagnóstico. Dos conceptos psicológicos denominados significado o sentido de la vida (meaning in lifel sense of life) y significado o sentido de la muerte (meaning of death/sense of death) son dos de los referentes teóricos de este estudio cuyo objetivo es comprender el significado de la vida y de la muerte en mujeres colombianas diagnosticadas con cáncer de mama y se encuentran en tratamiento oncológico, desde una perspectiva cualitativa.

En Colombia, el cáncer de mama cobra anualmente la vida de muchas mujeres. Su incidencia es de 30 por cada 100000 , hecho que ha aumentado la mortalidad en las colombianas y se ha convertido en un problema nacional (Piñeros et al., 2011). Además, en Colombia este es el cáncer de mayor incidencia en mujeres y la segunda causa de muerte en este país después del cáncer de cuello uterino (Pardo \& Cendales, 2010). De acuerdo con Pardo y Cendales, la mortalidad por este tipo de cáncer en el sexo femenino ha venido en aumento. Este es un hecho lamentable cuando se trata de un cáncer con una alta probabilidad de ser prevenido. Sin embargo, Piñeros et al. (2008) resaltan que el $60 \%$ de los diagnósticos de cáncer de mama que se diagnostican en Bogotá están en estado avanzado. A esto, Piñeros et al. (2008) añaden que son bajas las probabilidades de que la mujer con cáncer de mama reciba atención oportuna en el sistema de salud colombiano; reportan que en Bogotá, más de la mitad de las mujeres con este tipo de cáncer esperan cerca de tres meses entre la primera consulta y el inicio del tratamiento oncológico.

Murillo et al. (2008) plantean que mientras el cáncer de cuello uterino es característico de mujeres de zonas pobres del país; el cáncer de mama lo es de mujeres que no se encuentran en condición de pobreza, que pertenecen al régimen contributivo del sistema de salud colombiano, lo que implica que aportan dinero para recibir servicios de salud y que los cálculos de años de vida potencialmente perdidos sean diferentes según el régimen de vinculación al sistema. El Plan Decenal para el Control del Cáncer en Colombia 2012-2021 (2012) espera lograr que antes del 2021 se detecte en estadios tempranos el $60 \%$ de los casos de cáncer de mama. En tanto esto se logra, la realidad en Colombia es 
alarmante frente a la morbimortalidad por este tipo de cáncer. La probabilidad de que una mujer colombiana muera por este cáncer es alta y, por consiguiente, su diagnóstico se convierte en un evento amenazante que, entre otros, puede llevar a reflexionar sobre el significado de la vida y de la muerte. No hay duda de que la detección temprana del cáncer de mama es una meta fundamental en Colombia; pero mientras esto es una realidad, la atención integral de estas mujeres, desde el diagnóstico hasta el desenlace de la enfermedad, es igualmente fundamental. De hecho, así se reconoce y se ha propuesto como acción comunitaria en el Plan Decenal para el Control del Cáncer en Colombia 2012-2021 (2012).

Con base en esta realidad, ¿qué significan la vida y la muerte para las mujeres con cáncer de mama? Para responder a esta pregunta se tomó como marco de referencia teórico la teoría de las representaciones sociales (TRS), de Serge Moscovici. De acuerdo con este autor, las representaciones son básicamente un "sistema de clasificación, denotación y asignación de categorías y nombres" (Moscovici, 1984, p. 30). Según la fuerza y las características de las representaciones sociales, estas se transmiten de generación en generación, al punto de que un conocimiento popular llega a ser compartido por millones de personas (Moscovici, 1998). Es decir, una representación social acerca de determinado objeto puede estar en cualquier persona, discriminando su nivel educativo, etnia, religión o condición física, y en este punto se hace evidente que, por extraño que llegue a resultar el conocimiento popular, tiene algo que mostrar, puesto que enseña cómo y qué piensa la gente sobre ciertos fenómenos, además de exponer su preferencia por el conocimiento popular antes que por el conocimiento científico (Moscovici, 1998).

Hay antecedentes de investigaciones que abordan las representaciones sociales del cáncer de mama. Es el caso del estudio cualitativo de Giraldo-Mora y Arango (2009), dirigido a comprender las representaciones sociales del cáncer de mama y cómo estas influyen en las conductas de prevención y de autocuidado en mujeres sin cáncer. Estos autores encontraron que los senos tienen una representación centrada en el atractivo de la mujer; pero, a su vez, representaciones sociales negativas que afectan también negativamente la prevención y el autocuidado.

Giraldo-Mora (2009) realizó un estudio cualitativo en la ciudad de Medellín (Colombia) con 19 mujeres sin cáncer. El autor en mención encontró que el cáncer se considera una enfermedad destructiva y catastrófica que genera sufrimiento y afecta la integridad del cuerpo. Estas mujeres también consideraron que el cáncer de mama tiene efectos psicológicos, sociales y fisiológicos y cuya etiología está en problemas afectivos, personales y hábitos no saludables.

El Ministerio de Salud de Argentina y el Instituto Nacional del Cáncer de ese mismo país realizaron un estudio cualitativo, nacional, dirigido - entre otros propósitos - a explorar las representaciones socioculturales sobre el cáncer, en general, y sobre el cáncer de cuello uterino, colon y mama, en particular (Ramos et al., 2013). Entre otros, justifican su estudio en que en Latinoamérica los estudios sobre las representaciones sociales del cáncer son escasos. También hicieron este estudio con mujeres sin cáncer, bajo el argumento de que se necesita su visión para realizar actividades de prevención más efectivas. Hallaron que las representaciones que tienen del cáncer de mama es que se encuentra dormido y cuando algo, que no saben qué es (las ideas pasan por los golpes, el estrés, la promiscuidad, etc.), lo despierta, invade los senos y les causa cambios morfológicos notorios, y como todos los demás tipos de cáncer, tiene la capacidad de metastatizar.

Sin embargo, no se encontraron antecedentes que exploren el significado o el sentido de la vida y de la muerte para las mujeres que desarrollan este tipo de cáncer. Precisamente, la TRS "también enfatiza la importancia de los significados; 
el papel de los aspectos simbólicos y de la actividad interpretativa de las personas" (Araya, 2002, p. 19).

Así mismo, a pesar de que hay pocos registros sobre estudios relacionados con el tema en cuestión, hay investigaciones que retoman similitudes en aspectos temáticos y metodológicos. Parra, García e Insuasty (2011) realizaron un estudio similar en población colombiana, que comparte aspectos de la metodología usada en la presente investigación. Si bien el foco de cada uno de los estudios es distinto, ambos se centran en la experiencia de las mujeres con cáncer de mama y en cómo el afrontamiento de esta enfermedad modifica la concepción y la manera en que las pacientes viven su vida después de recibir el diagnóstico.

\section{El significado (sentido) de la vida}

De acuerdo con Kállay (2008), es una tendencia inherente a la especie humana atribuir significado a la mayoría de los eventos cotidianos. En particular, con respecto al significado de la vida, Ho, Cheung y Cheung (2010) indican que usualmente se ha referido al sentido de coherencia o al entendimiento de la existencia y se asocia con que las personas tengan un propósito en la vida. Según Wong y Fry (1998), se trata de un constructo individual y un sistema cognitivo basado en el marco cultural de la persona, que influencia su toma de decisiones, actividades y metas; es un propósito, un valor personal y un sentido de realización. De esta manera, esta experiencia de encontrar el sentido permite a las personas trascender las experiencias intrusivas $\mathrm{y}$ negativas, que promueven una vida positiva $\mathrm{y}$ saludable.

Según Sorajjakool y Seyle (2005), la necesidad de sentido es inherente al ser mismo, es trascendental a la existencia, y esto se hace evidente cuando es necesario enfrentar una situación de crisis, por ejemplo, una enfermedad crónica como el cáncer. De hecho, el sentido de la vida está asociado tanto con recursos cognoscitivos de las personas como con significados y propósitos (Bering, 2003). Dar un sentido positivo a la enfermedad es principalmente cognitivo. La persona enferma puede evaluar o aceptar la enfermedad ya sea como una posibilidad de crecer y cambiarse a sí mismo o como una restricción en la vida (Butkutè \& Perminas, 2005). Sin embargo, la subjetividad del constructo sentido de la vida ha generado variaciones en su definición (Ho et al., 2010). No obstante, se reconocen algunos elementos esenciales en este (Mischel \& Shoda, 1995): (a) es un rasgo que depende de la personalidad de las personas, (b) es un elemento cognitivo y (c) debido a su subjetividad, difiere de persona a persona. Fegg et al. (2010) recomiendan definir el significado de la vida en términos de comportamientos y circunstancias que con frecuencia son fuentes de sentido, como el crecimiento personal, las relaciones sociales y las contribuciones que las personas hacen a la sociedad. Por su parte, Wong y Fry (1998) proponen la idea que existen diferentes fuentes por las que una persona puede experimentar darle sentido a la vida, y Jaarsma, Pool, Ranchor y Sanderman (2006) distinguen tres componentes: (a) cognitivo: creencias y diferentes interpretaciones del mundo; (b) emocional: luchas, dificultades y los logros personales, y (c) afectivo: sentimientos de realización y satisfacción con la vida.

Por su parte, Kállay y Miclea (2007) plantean que encontrar sentido a la vida contribuiría al proceso de adaptación, el cual, en el caso de los pacientes diagnosticados con una enfermedad amenazante, es importante y contribuiría, a su vez, a una mejor calidad de vida.

Desde la experiencia clínica, las investigaciones sistemáticas y las anécdotas populares, es claro que una vez la persona es confrontada con la muerte (propio del impacto diagnóstico por una enfermedad grave, crónica o mortal), cambia el criterio con el cual ha evaluado su vida (Salmon \& Valori, 1996). De hecho, la percepción de satisfacción con la vida en pacientes con cáncer con pronóstico desfavorable difiere de la percepción que tienen las personas que se encuentran en condiciones de salud óptimas (Salmon \& Valori, 1996). Las anécdotas 
populares también sugieren cambios en las cogniciones y actitudes de las personas, pues aquellas que se confrontan con la muerte, debido a una enfermedad como el cáncer, describen su vida como menos problemática, con menos estrés y aprecian la vida más que antes de tener un diagnóstico desfavorable (Wong \& Fry, 1998).

A partir de la interpretación constante de los eventos vitales, la persona le otorga un sentido positivo o negativo a la vida. Las personas que encuentran un mayor nivel positivo de sentido a sus vidas y frecuentemente tienen mejores mecanismos específicos de afrontamiento adaptativo, de aquellas personas que tienen un menor nivel. Estas personas tienen una tendencia frecuente de reinterpretar positivamente los eventos vitales (Kállay \& Miclea, 2007). De acuerdo con Taylor (1993), en caso de enfermedad, la adaptación a ella también está relacionada con un sentido positivo de la vida y una mejor calidad de vida.

Así mismo, estudios como los de Reker, Peacock y Wong (1987); Zika y Chamberlain (1992), y Jaarsma et al. (2006) refieren que encontrar el sentido de la vida propia está positivamente relacionado con percepción de bienestar general, manifestado por medio de habilidades como la autoeficacia, los diferentes mecanismos de afrontamiento, los diferentes grados de religiosidad, el apoyo social, entre otras características, que dan cuenta de la construcción positiva del sentido.

Kállay y Miclea (2007) encontraron que las personas que no están en capacidad de encontrar un sentido positivo para su vida tienden a la falta de compromiso y a la pasividad, lo cual evidencia un comportamiento desadaptativo en el afrontamiento de situaciones difíciles.

Es importante resaltar que para los pacientes con enfermedades graves como el cáncer, el sentido de vida y el significado que tienen de sí mismos se reorganizan con base en la situación que enfrentan (Sherman, Simonton, Latif \& Bracy, 2010). Sin embargo, es necesario admitir que la relación existente entre una enfermedad crónica y la forma como la persona afronta tal enfermedad, es subjetiva y depende de diversos factores, entre los que se destacan: el curso de la enfermedad, las circunstancias en las que la persona se encuentra (ya sean sociales, económicas, familiares, etc.), la edad y otros elementos particulares del ámbito psicológico de quien tiene la enfermedad (Butkutè \& Perminas, 2005).

Específicamente, en el caso del cáncer de mama, Butkutè y Perminas (2005) describen que el proceso de afrontamiento de la enfermedad también se ve reflejado en la manera como la persona la significa. De hecho, de acuerdo con Anagnostopoulos, Slater, Fitzsimmons y Kolokotroni (2011), el sentido de la vida media el impacto psicológico de los pacientes con cáncer de mama, pues está muy relacionado con bajos niveles de malestar emocional. Por ejemplo, cuando la mujer tiene cáncer, puede emplear diferentes estrategias para afrontar activamente la enfermedad y verla entonces como un desafío. Tal estrategia es una manera de afrontar la enfermedad, pelear contra ella, buscar información para promover el control y orientar el problema para superarlo (Livneh, 2000).

Sin embargo, la enfermedad también puede verse como un enemigo, de modo que la persona se siente sin fuerzas para afrontarla (Beaver et al., 1996). Puede llegar a sentirse ansiosa, deprimida e, incluso, expresar sentimientos y comportamientos hostiles. Entender la enfermedad de esta manera o entenderla como un castigo, una debilidad o una pérdida de posibilidad hace que la persona tenga sentimientos de pena o vergüenza, pérdida de control y depresión. Así, estas últimas pueden considerarse las estrategias menos adaptativas para afrontar la enfermedad (Butkutè \& Perminas, 2005).

Beaver et al. (1996) proponen que si la enfermedad se ve de manera positiva, es porque es entendida como: (a) un alivio, por cuanto significa una oportunidad para cambiar de rutina; (b) un valor, ya que tanto la enfermedad como el sufrimiento que esta conlleva se piensan como un don al que se le debe dar un significado positivo, y (c) una estrategia, 
donde la enfermedad es la oportunidad de recibir beneficios secundarios como protección o atención.

En otro estudio realizado por Rustoen y Begnum (2000) se encontró que aquellas mujeres con cáncer de mama que decidieron adoptar un espíritu de lucha mostraron mejorías sin recurrencia en los siguientes cinco, diez y hasta quince años después del diagnóstico, en contraste con aquellas cuya posición fue de fatalismo e impotencia. Entre mayor sentido de significado tenga la paciente con cáncer de mama, presentará mayor cohesión social y psicológica y mayor ajuste emocional (Ramfelt, Severinsson \& Lutzen, 2002; Schnoll, Knowles \& Harlow, 2002). También se ha encontrado que el sentido de vida positivo contribuye a una disminución en la fatiga (Thompson, 2007), uno de los principales y de los más incapacitantes síntomas presentados por estas mujeres.

La manera como se ve la vida y como se evalúa es de suma importancia, pero también es relevante conocer por medio de qué elementos las personas dan el sentido respectivo a su vida. En un estudio de Arman y Backman (2007) se identificó que para algunos pacientes con diagnóstico de cáncer: "todo tiene sentido, la vida entera, incluyendo las actividades ordinarias de la vida diaria" (p. 447). Lo anterior, refiriéndose a "las opiniones espontáneas dadas por los participantes donde el simple hecho de vivir hace que todo en su vida se vuelva más importante. Así mismo, las relaciones con la familia, amigos y personas traen significado a la vida" (p. 447), ya que la calidad de las relaciones y el tiempo invertido en ellas ayuda a la construcción de significado.

La enfermedad, al ser una condición subjetiva y diferente en cada uno de los pacientes afectados, permite la diversificación de valoraciones y significados, tanto para su enfermedad como para su vida en general. El cáncer como enfermedad crítica puede cambiar radicalmente las condiciones del paciente, lo cual permite que los sentidos de vida, muerte, enfermedad y oportunidad se vean reformados y se acomoden a la nueva situación. Lo anterior permite concluir que los significados son variables y se acomodan a las limitaciones o posibilidades presentes en la persona.

Pese a las múltiples motivaciones o referentes que toman las pacientes para continuar con sus vidas y luchar contra la enfermedad, existe evidencia del gran impacto que sobre ellas tienen, la vulnerabilidad (temor a la recaída) y el significado positivo (cambios positivos en las creencias, percepciones y comportamientos ocurridos, debido a la experiencia con el cáncer) que, de no manejarse adecuadamente, pueden llegar a mucho malestar emocional y pobre ajuste global (Bower et al., 2005).

De acuerdo con lo anterior, es probable que una enfermedad como el cáncer modifique tanto el significado del sentido de la vida como el de la muerte.

\section{EI significado (sentido) de la muerte}

Las experiencias personales con la muerte permiten renovar el valor de la vida (Marcu, 2007), es decir, luego de confrontar la finitud y la fragilidad natural de lo que es la vida, el valor o la apreciación de las personas cambia sustancialmente, hasta el punto de darle mayor importancia (King, Hicks \& Abdelkhalik, 2009).

Si bien la interpretación del sentido de la vida y el sentido de la muerte es subjetiva, existen dinámicas sociales y culturales que comparten información que ayuda al individuo a crear un propio sentido de muerte, el cual puede ser común (Marcu, 2007). Estas dinámicas y experiencias que ayudan a la producción de significado pueden variar y, así mismo, resignificar el sentido de muerte durante el transcurso de la vida del individuo (Marcu, 2007).

Para Byock (2002), los seres humanos se enfrentan a diversos problemas y lo esperable es que estas situaciones se puedan resolver; pero cuando no es así, tanto la cultura como el individuo asignan un significado a este problema sin resolución. El tipo de significado que la persona dé (es decir, positivo o negativo) a un evento como la muerte tiene un impacto en las consecuencias o las características para su vida y serán similares a la forma 
de entender el mundo y la forma de enfrentarse, por ejemplo, a una enfermedad (Cicirelli, 1998).

Es pertinente identificar que el significado de vida y muerte son interdependientes, por cuanto la vida cobra un significado por el hecho, el evento y la existencia de la muerte. Y de la misma manera como la persona vive, las metas que logra, las fallas, los esfuerzos, los obstáculos sobrepasados, entre otros elementos, son los encargados de significar la vida (Cicirelli, 1998). Byock (2002) expone que confrontar a la muerte hace que las personas se cuestionen existencialmente y que, a raíz de estas experiencias, se forjen significados en torno al sentido de vida y de muerte.

Feifel, citado por Cicirelli (1998), reporta que solo el hombre es quien tiene la capacidad de reaccionar emocionalmente al concepto objetivo específico de la muerte, al entender sus limitaciones en cuanto a lugar y tiempo, cesar las funciones corporales y saber que este es un proceso inevitable e irreversible para todos.

Mientras algunos autores como Byock (2002) han indicado que personas con creencias y prácticas religiosas arraigadas tienen mayor facilidad para lidiar con la enfermedad y la muerte, otros - como Kahoe y Dunn (1975) - han negado la relación entre religión y miedo a la muerte.

Pese a que la experiencia de muerte es universal e implica un cese de las funciones mentales y corporales, cada persona la vive de manera distinta, con base en su edad, creencias, sexo, experiencias previas y cercanas con la muerte y significado que le otorgue; por ejemplo, motivación, extinción, vida en el más allá, legado o propósito (Cicirelli, 2001).

También, Cicirelli (1998) especifica que si bien los significados que son atribuidos a la muerte pueden ser individuales y dados por las experiencias únicas de la persona, igualmente pueden ser compartidos por un grupo específico que tiene experiencias y conceptos similares. De hecho, Byock (2002) hace referencia a que las historias, los relatos y las narraciones de otros llegan a jugar un papel fundamental en la creación de significados en general.
Cuando se habla de sentido de muerte, no solo se trata el tema de la muerte o la pérdida, sino que también se habla de sufrimiento y duelo. Precisamente con respecto a este último, Byock (2002) indica que lo que causa ansiedad de la muerte, puede no ser la ausencia del ser, sino el dolor de la pérdida de haber sido.

Muchas veces, la interpretación de los objetos está relacionada con las reacciones emocionales que estos pueden provocar, de tal manera que si los significados atribuidos a la muerte son negativos, las reacciones pueden ser negativas, lo cual provocaría, por ejemplo, reacciones de miedo y temor (Cicirelli, 1998). Lo anterior se convierte en una dificultad, en cuanto el miedo es un complejo de emociones, no una emoción individual. La relación entre el significado que es dado a la muerte y el miedo que este significado provoca es la reacción emocional (Cicirelli, 1998).

Se supone que los significados de muerte deberían cambiar a lo largo de la vida y así mismo deberían hacerlo los miedos. Lo anterior es posible afirmarlo, dado que varios estudios han demostrado que los adultos mayores tienen menos miedo a la muerte y que los jóvenes tienen menos miedo hacia los eventos inesperados (Neimeyer \& Moore, 1994, citados por Cicirelli, 1998). De hecho, estudios como el de Thorson y Powell (1988) han mostrado que existen diferencias de género en la experimentación de la ansiedad con respecto a la muerte, al encontrar que las mujeres sentían mayor ansiedad a la muerte.

De acuerdo con Byock (2002), desde el momento en que una persona es diagnosticada con una enfermedad incurable, la muerte se convierte en una alarma continua que no "para de sonar", y aun cuando el paciente entre en estado de remisión o en un periodo relativo de salud, la muerte y la intrusión de pensar en la muerte lo fuerzan a mirar y a enfrentar cuanto quiere evadir. Estudios como el de Wilde y Murray (2009) han revelado que las experiencias directas con la muerte crean contextos y significados en cada persona y en su historia individual. Esta postura difiere de la creencia que 
se tenía antiguamente, cuando las experiencias conectadas a la muerte eran un episodio aislado, que generaba una crisis específica, pero que no se relacionaba con la concepción de vida y de muerte en las personas. De acuerdo con estos autores, las personas escogen ciertos elementos de cada una de sus experiencias, de situaciones que subjetivamente tienen un nivel de significancia más alto y aplican la interpretación de estas situaciones a su vida. Sucede igual con las experiencias cercanas a la muerte: la interpretación que las personas den a esta experiencia se va a incluir tanto en el sentido de vida como en el sentido de muerte.

Es evidente, entonces, que la muerte es un elemento esencial de la vida, que hace parte únicamente de la conciencia humana y que es un constructo elaborado personalmente, pero que se comparte de modo cultural y social, lo cual le otorga un valor importante en la existencia humana.

En cuanto al significado de la vida y de la muerte en mujeres con cáncer de mama, Thompson (2007) realizó un estudio correlacional dirigido a establecer las relaciones entre el sentido de vida, la fatiga y el malestar emocional en un grupo de mujeres supervivientes de este tipo de cáncer. Por su parte, Meraviglia (2006) encontró que el sentido de vida es un elemento de la espiritualidad y en su estudio buscó examinar los efectos de esta (sentido de la vida y oración) sobre el sentido de bienestar en mujeres supervivientes de cáncer de mama.

Por otra parte, Jim y Andersen (2007) condujeron dos estudios con el propósito de identificar si el sentido de la vida puede actuar como mediador entre la funcionalidad física y social y el malestar emocional en pacientes con cáncer. Si bien la muestra fue de pacientes con diversos tipos de cáncer, predominaron aquellos con cáncer de mama.

No se encontraron estudios específicos del sentido de la muerte en mujeres con cáncer de mama, ni estudios de corte cualitativo que aborden el significado de la vida y de la muerte para este grupo poblacional. Aún menos, estudios con población latinoamericana y específicamente colombiana.

\section{Objetivos}

El objetivo general de este estudio fue comprender el significado de la vida y de la muerte para mujeres con cáncer de mama. Adicionalmente, se plantearon como objetivos específicos: (a) describir las representaciones sociales de las pacientes sobre la vida y la muerte y (b) analizar la experiencia de las pacientes frente al diagnóstico y el tratamiento identificando los significados de la vida y de la muerte en estos momentos de la enfermedad.

\section{Método}

\section{Tipo de investigación}

El presente fue un estudio cualitativo, exploratorio.

\section{Población y muestra}

La población de estudio fueron mujeres adultas con cáncer de mama en tratamiento de quimioterapia. La muestra se obtuvo por conveniencia hasta saturar los datos. Un total de once mujeres conformaron la muestra definitiva; su promedio de edad fue de 55,9 años (la menor fue de 23 años y la mayor de 68 años). Siete de ellas estaban casadas, tres eran solteras y una era viuda. Nueve de ellas reportaron haber sido diagnosticadas con cáncer de mama en los últimos 3 años; nueve de ellas en estadio III y dos en estadio II. Seis de ellas reportaron haber sido mastectomizadas.

\section{Instrumentos}

Se utilizó como principal fuente de recolección de información una entrevista semiestructurada, diseñada para promover la narrativa de las pacientes e indagar la experiencia vivida con el cáncer, con la quimioterapia, la forma en que esa experiencia ha afectado su vida, los cambios que en ella ha generado, el afrontamiento, los pensamientos 
sobre la muerte, sobre el suicidio, entre otros. Así mismo, se utilizó una ficha de información general en la que se indagó por datos de tipo sociodemográfico.

\section{Procedimiento}

Inicialmente, se informó a las pacientes sobre la posibilidad de participar en la investigación y luego de esta exposición y aceptación de las mujeres, se procedió a firmar el consentimiento informado de cada una de las pacientes tanto para la participación voluntaria en la investigación como para la grabación en audio de la entrevista. Así mismo, se les presentaron los objetivos de la investigación, las molestias o riesgos esperados que podían generarse por la entrevista y, además, los beneficios que recibirían por su participación en el estudio. De igual manera, se insistió en el carácter confidencial de la información proporcionada. Se resolvieron las dudas e inquietudes que emergieron durante su lectura. Una vez obtenido el consentimiento, se registraron los siguientes datos generales: edad, estado civil, estrato socioeconómico, lugar de nacimiento, lugar de procedencia, religión, realización de prácticas religiosas, fecha del diagnóstico, diagnóstico, estadio, cirugía oncológica y esquema de quimioterapia. Finalmente, se procedió a la entrevista semiestructurada, en la que se invitaba a la paciente a narrar su propia experiencia con el cáncer y que duró cerca de una hora. El entrevistador tuvo especial precaución de no emitir juicios de valor, de permitir y promover la narrativa y de atender a que los diversos aspectos de interés fueran retomados en caso de que se requirieran mayores detalles pertinentes y relevantes para el alcance de los objetivos de investigación propuestos. Todas las entrevistas se realizaron en el contexto hospitalario, en este caso el contexto natural para la paciente con cáncer de mama, pues es donde recibe la quimioterapia, es atendida en las citas médicas, es examinada y eventualmente es hospitalizada.

\section{Análisis de los resultados}

El análisis de los resultados de este estudio es de tipo cualitativo. Inicialmente, se realizó la codificación y la categorización de la información obtenida a partir de las entrevistas. Esta actividad se realizó con el apoyo del programa Atlas.ti. El análisis de toda la información fue el resultado de un proceso de triangulación en el que los cuatro investigadores analizaron de manera individual cada una de las once entrevistas transcritas, codificándolas, categorizándolas e interpretándolas, para luego poner en común el producto de este análisis y generar una versión definitiva. Finalmente, toda la información fue organizada en dos secciones distintas pero complementarias, a saber: (a) análisis descriptivo y (b) análisis interpretativo, según la sugerencia de Bonilla-Castro y Rodríguez-Sehk (2000).

En un principio, se realizó la categorización inductiva con base en las entrevistas realizadas, la cual originó varias categorías y subcategorías emergentes. La codificación y categorización se realizó entre los cuatro investigadores por medio de Atlas.ti. Las categorías emergentes fueron: apoyo, qué es el cáncer, tratamiento, efectos secundarios de los tratamientos oncológicos, suicidio, afrontamiento, experiencia positiva con la EPS, el médico y el hospital, reacciones emocionales, conocimiento previo de la quimioterapia, experiencias previas con el cáncer de personas conocidas o familiares, proceso de diagnóstico y médico como poseedor de conocimiento.

Posteriormente, las categorías emergentes se describieron e ilustraron con algunas de las citas provenientes del discurso de las pacientes entrevistadas, y se elaboraron algunas matrices descriptivas. Un ejemplo de ello puede observarse en la tabla 1, en la cual se hace evidente que el cáncer está representado de tal manera que, sin duda alguna, afecta el significado de la vida y de la muerte para estas mujeres. A partir de esta tabla 1 es posible conocer lo que para cada una de las entrevistadas es el cáncer, lo cual es relevante en el momento de 
determinar si esa definición que ellas tienen influye en su forma de percibir la vida y la muerte.

Tabla 1

Descriptores y citas de la categoría: ¿qué es el cáncer?

\begin{tabular}{|c|c|}
\hline Descriptores & Citas \\
\hline 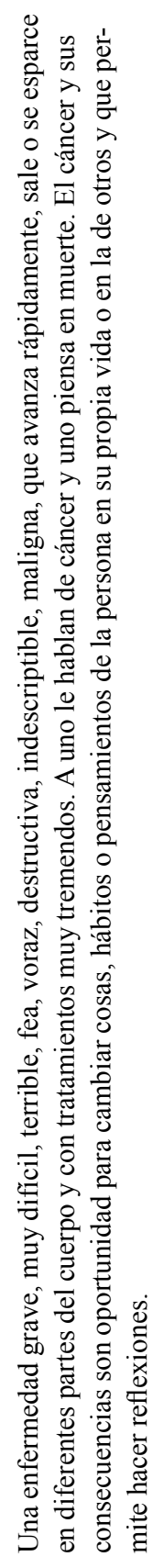 & 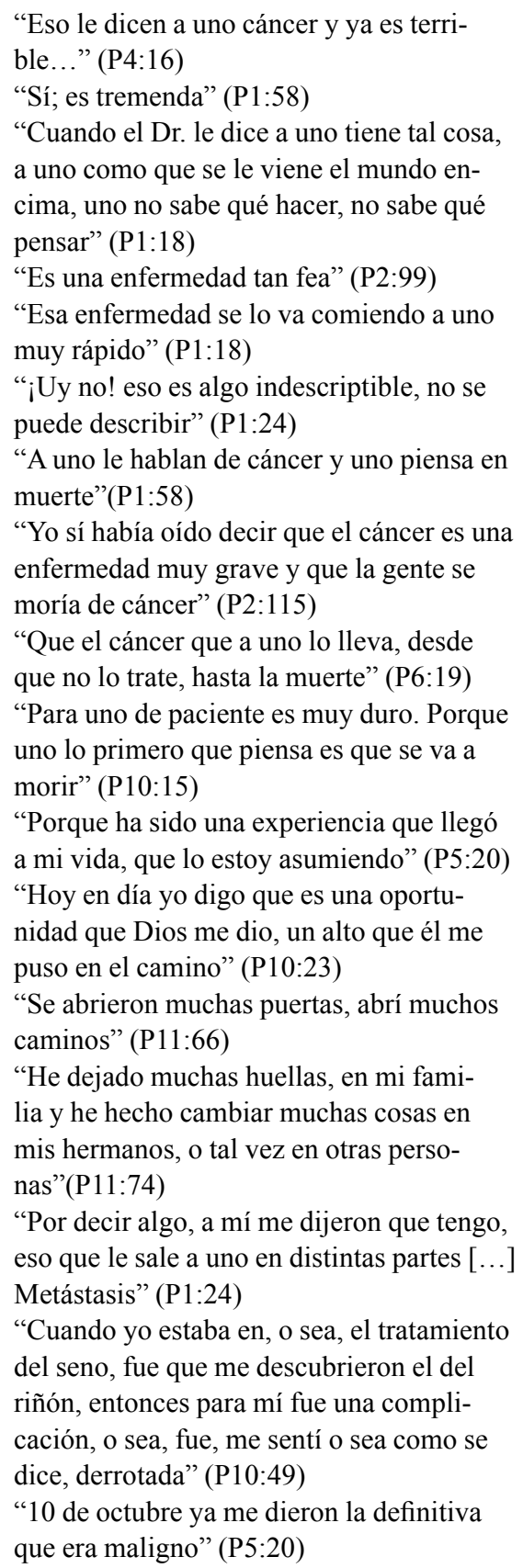 \\
\hline
\end{tabular}

Por último, como parte del análisis descriptivo, se elaboraron taxonomías con el objetivo de clasificar de manera más detallada la información contenida en algunas de las subcategorías presentadas, las cuales fueron representativas en el análisis inductivo. Así mismo, se elaboró una cadena lógica basada en la descripción del proceso de diagnóstico.

\section{Aspectos éticos}

El presente estudio fue sometido y aprobado por el Comité de Ética del centro oncológico privado al que asistían las pacientes. En coherencia con la Resolución 008430 de 1993, "por la cual se establecen las normas científicas, técnicas y administrativas para la investigación en salud", en este estudio se respetaron todos los principios de la bioética y se cuidó especialmente de respetar la confidencialidad de la información proporcionada por las participantes quienes participaron de manera voluntaria. Además, se obtuvo el consentimiento informado de cada una de ellas y las grabaciones se guardaron en la oficina del investigador principal, en archivo digital, al igual que la copia de los consentimientos informados.

Finalmente, se realizó el análisis interpretativo de los datos y se elaboró un esquema comprensivo de las representaciones sociales de la vida y de la muerte en la mujer con cáncer de mama. Dicho esquema se fue interpretando y se utilizaron algunas citas de lo expresado por las participantes como respaldo de tales interpretaciones.

\section{Resultados}

\section{Características de la población}

En lo referente a las características de las participantes, todas indicaron ser del régimen contributivo, lo que significa que pagan mensualmente al Estado su vinculación al Sistema General de Seguridad Social en Salud. Todas ellas viven en la ciudad de Bogotá, la mayoría en los estratos 2 y 3 , lo cual implica que pertenecen a los estratos socioe- 
conómicos más bajos y son personas que cuentan con escasos recursos, por lo cual reciben subsidios por parte del gobierno para cubrir los gastos de los servicios públicos domiciliarios (Departamento Administrativo Nacional de Estadística, s. f.). En Colombia, el cáncer es considerado una enfermedad de alto costo cuyo diagnóstico y tratamiento son en su totalidad cubiertos por el Estado. Por ser el tratamiento oncológico especializado y de alto riesgo, se presta en instituciones hospitalarias de alto nivel de complejidad (tercer y cuarto nivel). ${ }^{1}$ Las mujeres que participaron en el estudio eran pacientes activas de una Institución Prestadora de Servicios de Salud (IPS) de tercer nivel, de carácter privado.

\section{Análisis descriptivo}

En el análisis de resultados emergieron varias categorías asociadas con el significado de la vida y de la muerte para las mujeres participantes (tablas 2 a 4). Aquellas asociadas con el significado de la vida fueron el apoyo social (que puede ser de diversos tipos: familiar, general, del equipo médico tratante o espiritual). Específicamente, se resalta la importancia del apoyo familiar y espiritual para estas mujeres.

El afrontamiento es una categoría que presenta ambivalencia; algunas estrategias asociadas con el sentido de vida y otras con el sentido de muerte. Sorprende que la intención de lucha contra la

Tabla 2

Categorías y subcategorías emergentes: apoyo; ¿qué es el cáncer?; tratamiento

\begin{tabular}{|c|c|c|c|}
\hline Categoría & Subcategorías & Código & Frecuencia \\
\hline \multirow{4}{*}{ Ароуо } & Apoyo familiar & 1 & 56 \\
\hline & Apoyo social & 2 & 26 \\
\hline & Apoyo del equipo médico tratante & 3 & 15 \\
\hline & Apoyo espiritual & 7 & 30 \\
\hline \multirow{12}{*}{$\begin{array}{l}\text { ¿Qué el } \\
\text { cáncer? }\end{array}$} & Cáncer como experiencia que cambia la vida & 4 & 23 \\
\hline & Cáncer como sinónimo de muerte & 106 & 10 \\
\hline & Temor a la enfermedad & 68 & 2 \\
\hline & Cáncer como algo terrible & 29 & 6 \\
\hline & Efectos físicos de la enfermedad & 33 & 1 \\
\hline & Cáncer como algo destructivo-voraz & 48 & 2 \\
\hline & Metástasis & 52 & 8 \\
\hline & Cáncer como algo indescriptible & 51 & 1 \\
\hline & Adenomegalias & 58 & 1 \\
\hline & Cáncer como generador de incapacidad & 59 & 7 \\
\hline & Cáncer como una enfermedad fea & 76 & 1 \\
\hline & Cáncer como prioridad. Es una prioridad tratarlo & 73 & 3 \\
\hline
\end{tabular}

1 "El cuarto nivel de complejidad es donde se atienden las enfermedades definidas como catastrófica, como el cáncer, el VIH-sida, las enfermedades cardiovasculares, trasplantes de órganos, insuficiencias renales crónicas, entre otras" (Gómez, 2007, p. 166). Para brindar la adecuada atención a enfermedades de mayor complejidad es necesario contar con unidades de cuidados intensivos de acuerdo con edad, laboratorios clínicos, imagenología, procedimientos especiales para garantizar el diagnóstico y el tratamiento y médicos especialistas (Gómez, 2007). 


\begin{tabular}{|c|c|c|c|}
\hline Categoría & Subcategorías & Código & Frecuencia \\
\hline \multirow{7}{*}{$\begin{array}{l}\text { ¿Qué el } \\
\text { cáncer? }\end{array}$} & Malignidad (tumor, enfermedad maligna) & 11 & 4 \\
\hline & El cáncer le puede dar a todo el mundo & 31 & 2 \\
\hline & Discriminación de otras personas hacia el paciente & 60 & 2 \\
\hline & El diagnóstico de cáncer genera sufrimiento al paciente y a otros & 28 & 3 \\
\hline & Aislamiento/soledad & 103 & 3 \\
\hline & Lástima & 80 & 2 \\
\hline & Cáncer que afecta varias áreas de la persona & 61 & 3 \\
\hline \multirow{9}{*}{ Tratamiento } & Cirugía/amputación & 22 & 16 \\
\hline & Tratamientos quimioterapia y radioterapia & 53 & 24 \\
\hline & Quimioterapia como algo fuerte & 55 & 21 \\
\hline & Quimioterapia como tratamiento inefectivo & 56 & 3 \\
\hline & Quimioterapia como tratamiento efectivo & 57 & 8 \\
\hline & Quimioterapia como martirio & 26 & 6 \\
\hline & Tratamientos caseros & 94 & 2 \\
\hline & Quimioterapia como procedimiento que disminuye el tamaño del tumor & 17 & 4 \\
\hline & Posibilidad de curación & 90 & 2 \\
\hline
\end{tabular}

Tabla 3

Categorias y subcategorias emergentes: muerte; efectos secundarios de los tratamientos oncológicos; afrontamiento y suicidio

\begin{tabular}{|c|c|c|c|}
\hline Categoría & Subcategorías & Código & Frecuencia \\
\hline Muerte & Pensamientos de muerte/deseo de morir & 104 & 10 \\
\hline \multirow{9}{*}{$\begin{array}{l}\text { Efectos } \\
\text { secunda- } \\
\text { rios de los } \\
\text { tratamientos } \\
\text { oncológicos }\end{array}$} & Dolor & 19 & 14 \\
\hline & Alopecia & 20 & 15 \\
\hline & Resequedad en la piel & 21 & 1 \\
\hline & Osteoporosis & 54 & 1 \\
\hline & Linfedema & 70 & 1 \\
\hline & Pesadez en los brazos & 71 & 1 \\
\hline & Náuseas/vómito & 100 & 6 \\
\hline & Boca con sabor a sangre & 101 & 1 \\
\hline & Mareo & 102 & 2 \\
\hline \multirow{5}{*}{$\begin{array}{l}\text { Afronta- } \\
\text { miento }\end{array}$} & Intención de lucha contra la enfermedad & 9 & 35 \\
\hline & Actitud de superación personal y de lucha & 10 & 34 \\
\hline & Negociar con Dios las experiencias de la enfermedad ("Dios da y Dios quita") & 32 & 15 \\
\hline & Los otros como un motivo para luchar & 38 & 20 \\
\hline & $\begin{array}{l}\text { Asimilación (ir incorporando las experiencias de la enfermedad a la vida-en- } \\
\text { tender la información) }\end{array}$ & 41 & 15 \\
\hline
\end{tabular}




\begin{tabular}{|c|c|c|c|}
\hline Categoría & Subcategorías & Código & Frecuencia \\
\hline \multirow{8}{*}{$\begin{array}{l}\text { Afronta- } \\
\text { miento }\end{array}$} & Autoinstrucciones & 42 & 8 \\
\hline & Anticipación de la enfermedad "Yo ya sabía" & 72 & 4 \\
\hline & Normalidad en la vida después del diagnóstico & 75 & 11 \\
\hline & Evitación de hablar de la enfermedad & 81 & 6 \\
\hline & Modificación de conductas debido a la enfermedad & 87 & 13 \\
\hline & Experiencias personales que influyen en el tratamiento & 93 & 4 \\
\hline & Reacción ante el diagnóstico & 47 & 13 \\
\hline & Ocultar el diagnóstico & 27 & 8 \\
\hline \multirow{6}{*}{ Suicidio } & Intención de abandonar/suspender el tratamiento de quimioterapia & 43 & 3 \\
\hline & Percepción de no control frente a la enfermedad y el tratamiento & 44 & 3 \\
\hline & Razones para el suicidio de personas con cáncer & 45 & 4 \\
\hline & Razones personales para el suicidio & 46 & 3 \\
\hline & Conocimiento del suicidio & 97 & 3 \\
\hline & Razones para no suicidarse & 98 & 4 \\
\hline
\end{tabular}

Tabla 4

Categorías y subcategorías emergentes: experiencia positiva con la EPS, el médico y el hospital; reacciones emocionales, conocimiento previo de la quimioterapia; experiencias previas con el cáncer de personas conocidas o familiares; proceso de diagnóstico y médico como poseedor de conocimiento

\begin{tabular}{|c|c|c|c|}
\hline Categoría & Subcategorías & Código & Frecuencia \\
\hline \multirow{3}{*}{$\begin{array}{l}\text { Experiencia positiva con } \\
\text { la EPS, el médico y el } \\
\text { hospital }\end{array}$} & Buena experiencia con la EPS & 62 & 3 \\
\hline & Buena experiencia con los médicos & 63 & 7 \\
\hline & Experiencia positiva con el hospital & 65 & 7 \\
\hline \multirow{3}{*}{ Reacciones emocionales } & Emociones negativas del paciente & 34 & 8 \\
\hline & Miedo a la quimioterapia & 24 & 4 \\
\hline & Experiencias personales negativas con la quimioterapia & 18 & 4 \\
\hline $\begin{array}{l}\text { Conocimiento previo de } \\
\text { la quimioterapia }\end{array}$ & & 25 & 9 \\
\hline $\begin{array}{l}\text { Experiencias previas con } \\
\text { el cáncer de personas } \\
\text { conocidas o familiares }\end{array}$ & & 8 & 28 \\
\hline \multirow{5}{*}{ Proceso de diagnóstico } & Crecimiento del tumor & 12 & 2 \\
\hline & Identificación de signos y síntomas & 13 & 13 \\
\hline & Lidiar con la tramitología y los obstáculos impuestos por el sistema de salud & 14 & 16 \\
\hline & Proceso de diagnóstico (exámenes, análisis, etc.) & 15 & 22 \\
\hline & $\begin{array}{l}\text { Tiempo transcurrido entre la aparición de signos y síntomas de la enfer- } \\
\text { medad y la emisión del diagnóstico }\end{array}$ & 16 & 6 \\
\hline $\begin{array}{l}\text { Médico como poseedor } \\
\text { de conocimiento }\end{array}$ & & 23 & 10 \\
\hline
\end{tabular}


enfermedad no es tan frecuente fue también una categoría que emerge en relación con el sentido de la vida. Predominan la intención de lucha contra la enfermedad; la actitud de superación personal y de lucha; los otros como un motivo para luchar; la asimilación que se consideró el proceso en el que las pacientes van incorporando las experiencias de la enfermedad a la vida y comprenden la información recibida y la negociación con Dios de las experiencias de la enfermedad ("Dios da y Dios quita"). Sin embargo, otros aspectos del afrontamiento están más asociados con el sentido de muerte: evitar hablar de la enfermedad, ocultar el diagnóstico por estar asociado con el sufrimiento de sí mismo y de otros y la reacción emocional negativa ante el diagnóstico. Algunas de las estrategias halladas parecen estar particularmente vinculadas con la necesidad de generar una percepción de control de un evento que se considera potencialmente mortal $\mathrm{y}$, por tanto, se encuentran vinculadas con el sentido de la muerte: la mujer sospecha que puede tener cáncer "Yo ya sabía que...". La figura 1 ilustra cómo están interrelacionados estos aspectos del afrontamiento.

Las experiencias positivas con el médico, el sistema de salud y el hospital hacen parte del sentido de vida, así como reconocer el valor de los conocimientos del oncólogo. A él es en quien finalmente recae la esperanza de la vida.

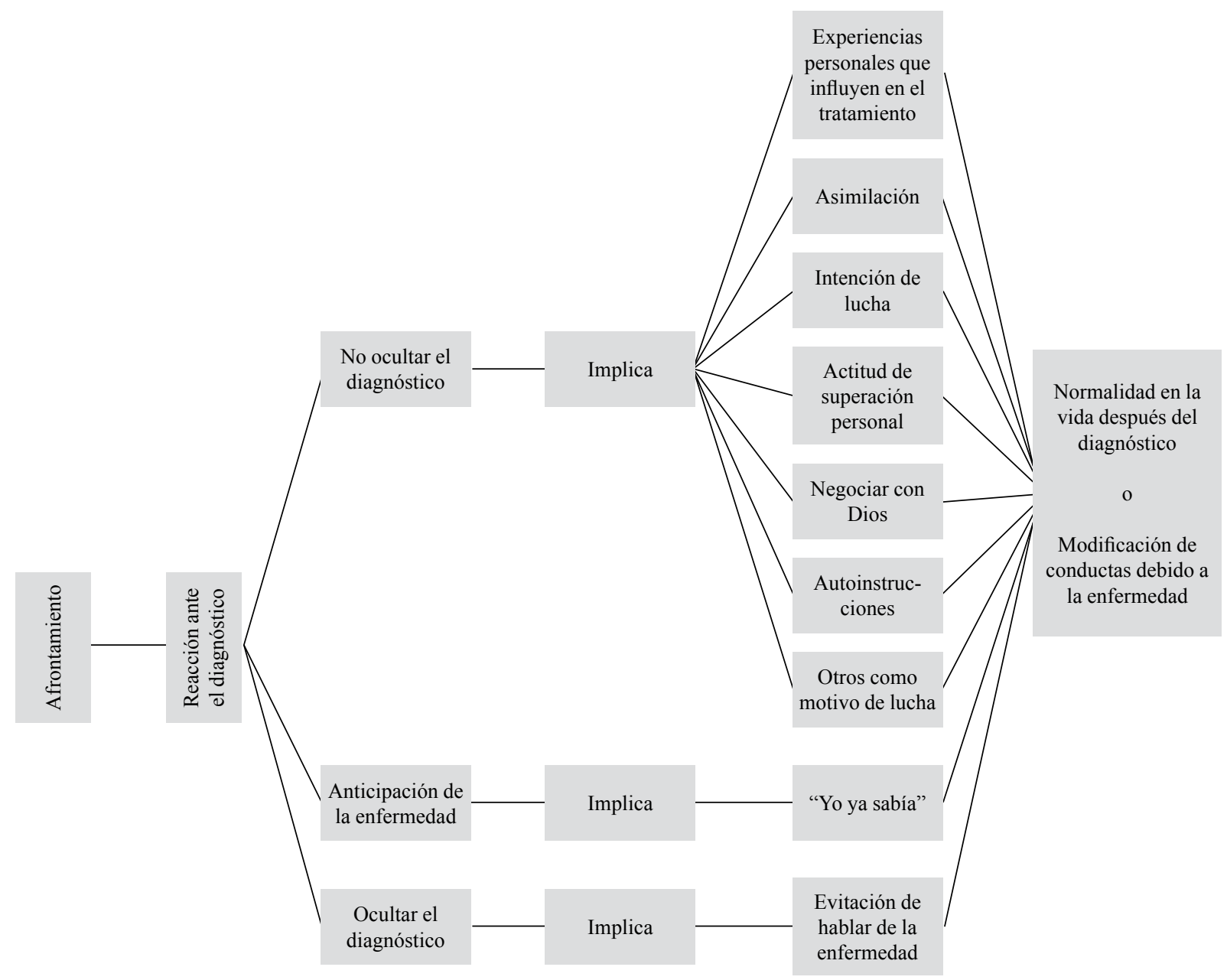

Figura 1. Taxonomía de afrontamiento 
Las demás categorías emergentes se vinculan con el sentido de muerte es negativo; es decir, le recuerdan al paciente que es mortal pero, además, que va a sufrir y a pasar por momentos difíciles durante el curso de la enfermedad. Es, en palabras de Byock (2002), una alarma continua. El cáncer se representa como una experiencia que cambia la vida; pero aunque esto pudiera considerarse positivo y relacionado con la vida, los discursos de las pacientes permitieron observar que se trata de una enfermedad cuyo sinónimo es la muerte, que genera temor, es terrible, tiene efectos en el organismo (por ejemplo, adenomegalias), es "destructivo-voraz", es maligno, requiere atención inmediata, tiene la capacidad de metastatizar, incapacita, es feo, indescriptible, genera sufrimiento al paciente y a otros, aísla o provoca lástima hacia el paciente. El tratamiento y sus efectos secundarios, la muerte, el suicidio, el miedo, entre otras, fueron otras de las categorías emergentes también vinculadas con el significado de la muerte.

Se observa que el proceso de diagnóstico inicia de dos maneras, ya sea por la identificación de signos y síntomas o por exámenes de rutina casualmente realizados. A partir de esto, se pasa por un proceso de tramitología con el sistema de salud, mientras que el tumor sigue con su crecimiento, como una sentencia de muerte para las mujeres que lo portan, hasta que finalmente se da el diagnóstico, pero ello no implica que la tramitología se detenga; el diagnóstico es solo otra etapa en el proceso que, sin duda, da lugar a nuevos trámites (figura 2).

\section{Análisis interpretativo}

Para las mujeres que participaron en este estudio, el cáncer de mama se concibe como algo inherente a ser mujer... un recordatorio que le anuncia a quien lo desarrolla que la vida es finita, que la muerte existe, que ahora... puede morir: "pues... la primer vez que pensé (en la muerte) fue el día que me dijeron que tenía cáncer en el seno" (P1:107) (figura 3).

¿Un evento? ¿Una prueba? ¿Una grave enfermedad? Se trata de cáncer, una enfermedad que "devora" y "consume" (P1: 107), que es capaz de migrar a otras partes del cuerpo; que pasa de ser algo inofensivo, muy eventualmente doloroso pero casi siempre indoloro, una "bola" o "una masa", a ser un "tumor maligno", de esos que mata.

Encontrarlo suele ser una casualidad y la evidencia de su presencia, un evento alarmante que precipita la búsqueda de una explicación que, en el fondo... suele anticiparse como negativa. Desde ese momento aparece en la mujer un pensamiento de sospecha, intrusivo, molesto: ¿y si es cáncer?: “... me empezó un dolorcito acá encima (señalando el seno izquierdo) y yo me tocaba y me tocaba y sentía una bolita pero yo sentía esa bolita, no me imaginaba nada que fuera a ser eso..." (P1:17).

Es posible que lo anterior permita comprender que la sentencia de ese cáncer se enajene, se distancie, que se le dé un lugar en el cuerpo, como si este no fuera propio: existe pero no es mío; en consecuencia... no me mataría.

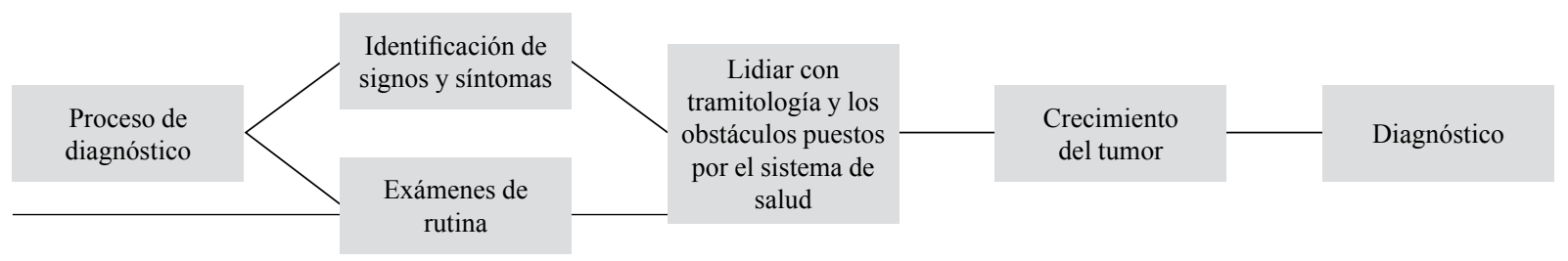

Figura 2. Cadena lógica del proceso de diagnóstico 


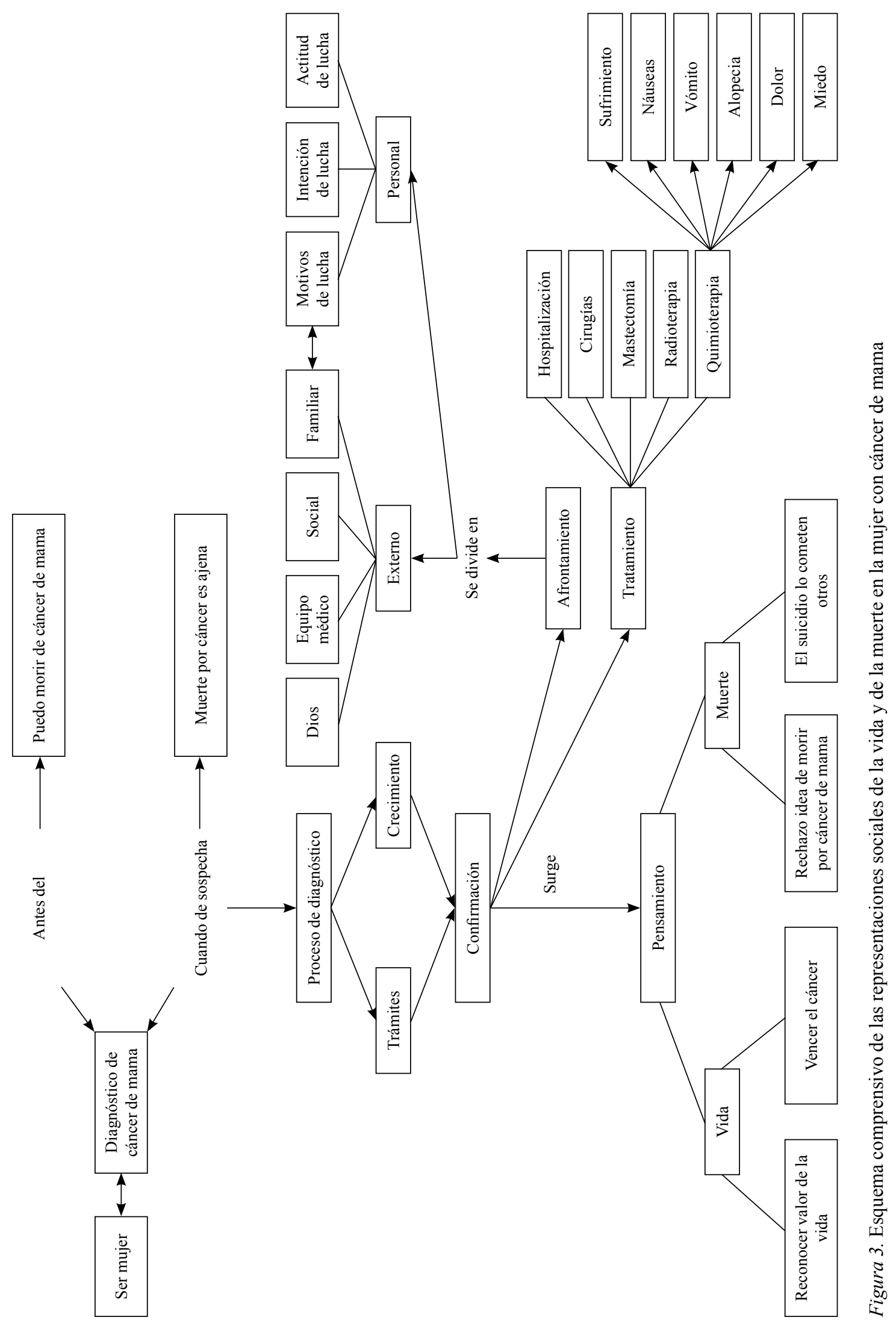


Una travesía para ser diagnosticada. Es eso lo que viven y relatan estas mujeres, el tiempo como elemento que juega en su contra; un minuto, unas horas, unos meses y el tumor crece sin control y les recuerda su autonomía. Autorizaciones, exámenes, citas, resultados y al final, la noticia: es cáncer:

Me hicieron un examen aquí en Bogotá en el L y me hicieron otro en Faca, dos veces ahí y al mes me hicieron los exámenes pero entonces empezaron una niña de Sasaima, empezó a trabar las cosas y no me daba las autorizaciones, me decía “es que yo estoy ocupada, no tengo tiempo"... Ese fue como mí, mi traba. Pero cuando ya se llegó así, yo dije no, háganme esto porque yo sé que tengo algo anormal en mi cuerpo, entonces fue cuando me hicieron la biopsia y entonces ya salió que era maligno. (P5:33)

Y, así, el diagnóstico de cáncer de mama se presenta como un estímulo que desencadena una respuesta de lucha intensa caracterizada por el ánimo y la actitud positivos; como si estos fueran definitorios en la victoria que se librará al alejar la presencia de reacciones emocionales consideradas negativas. Esa lucha implica un proceso de esfuerzos y resistencia contra ese cáncer que se personifica representándose como algo personificado que ahora vive dentro del seno, pero que si se trata de manera oportuna, terminará por desaparecer: "El que le daba ese mal, lo mataba y pues no, porque tratándolo uno a tiempo puede uno curarse" (P6:25).

¿Una oportunidad de vida? ¿Un posible escape de la muerte? Al parecer, el diagnóstico y su tratamiento le recuerdan a esta mujer que está viva y la obligan a desplegar recursos personales como su capacidad de afrontar y a reconocer recursos sociales como el apoyo de su familia, de sus allegados, de los miembros del equipo de salud e incluso y, por supuesto, de Dios: "La vida tiene que seguir adelante, luchar por mis nietos, por mi familia, por mi esposo, por mis hijas, por mi hijo...." (P5:153).
La religiosidad aparece de manera continua en los relatos de estas mujeres, como una especie de ancla que las estabiliza y le otorga a alguien más (un Dios) que a "ese cáncer", el poder de quitar la vida. Y a alguien más que está por encima de ellas, la capacidad de tranquilizarlas: “... gracias a Dios, Dios ha sido es el que me ha dado esa fortaleza para sobrevivir" (P1:7).

Precisamente, en lo que se refiere al afrontamiento. Estas mujeres relatan utilizar diversidad de estrategias que les permiten asimilar la enfermedad y llenarse de motivos que las animan a seguir adelante. Se trata: (a) de actitudes optimistas frente a la enfermedad, frente a la vida; (b) de intenciones de luchar activamente contra la enfermedad; (c) del reconocimiento de que existen otras personas como motivo para luchar; (d) de tener la capacidad de incorporar las experiencias de la enfermedad a la vida, y (e) muy eventualmente, de presentar conductas de evitación que no son necesariamente desadapativas.

Estas mujeres se fortalecen en sus razones para vivir y en la confianza que depositan en quién sabe cómo atacar el cáncer y salvarlas: el médico.

Estas mujeres saben que el tratamiento será intenso: hospitalizaciones, cirugías mutilantes, amputación del seno, de la cual solo se hace mención... sin lamentos. Así como tener cáncer de mama parece inherente a ser mujer, perder el seno en una cirugía, también lo es.

Además, puede predecirse que se recibirá quimioterapia. Aunque es un tratamiento del que se tiene información desde antes de tener el cáncer y se reconocen sus efectos secundarios, su representación es tan clara y tan negativa que atenúa (o prácticamente elimina) la radioterapia, que escasamente se enuncia en los relatos.

Quimioterapia es una experiencia que resulta terrible, dolorosa, dura, generadora de sufrimiento y miedo; pero que, según ellas, quieren creerlo, puede ser efectiva, porque es un tratamiento médico: 
... a pesar de que le da a uno como esa sensación de miedo de la quimio, el tumor me ha bajado mucho, bastante, bastante. (P5:49)

Uy, no, he sufrido tremendo. Eso es una cosa que le coge uno asco hasta la comida. Todo lo que uno toma, todo lo vomita, todo horrible, horrible. (P6:57)

Sí, tiene efectos secundarios, pero yo digo si, si en verdad sirve para algo no interesa que se la coloquen a uno. Si en verdad sirve para algo. (P1:46)

Pese a sus múltiples y molestos efectos secundarios, náuseas, vómitos y dolores que ahora ocupan un importante lugar en el discurso de la experiencia con la quimioterapia, es su única opción médica y si la rechazan, habrán perdido la batalla y demostrado una fragilidad que de manera explícita o tácita, ellas han decidido eliminar y remplazarla por un compromiso, también explícito o tácito, con su familia (en especial su pareja y sus hijos): dejar una huella a través de ser un ejemplo de mujer, de mamá. Quieren hacer de esta experiencia algo con sentido, una oportunidad de crecimiento personal, la oportunidad de ser más espiritual, de dejar una huella. Y, así, pueden sentirse satisfechas con su lucha:

... en este momento yo digo que ya he hecho, he dejado mucha huellas, en mi familia y he hecho cambiar muchas cosas en mis hermanos, o tal vez en otras personas, porque ellos eran muy duros, el hecho de entregar mi mamita a Dios, dejar a mis hijos, pues no con las profesiones que yo de pronto hubiera querido, pero ya dejarlos que se defiendan, darles ya cosas que yo diga bueno no van a sufrir porque les deje un estudio. (P11:74)

Estas mujeres reconocen que pueden morir, que a veces el sufrimiento es tan alto que los pensamientos sobre la muerte aparecen, con o sin permiso. Pero la estrategia está en combatirlos, alejar la muerte con los actos y con el pensamiento y, en especial, rechazar la idea de causarse la muerte, de suicidarse, aunque sea algo a lo que otros pacientes, no ellas, podrían sucumbir en el agotamiento causado por la enfermedad. Con la enfermedad y con el tratamiento, el cuerpo hace presencia, las sensaciones corporales les recuerdan que están vivas y paradójicamente, lo prefieren a la muerte:

Antes... pues cada nada pensaba que... muchas veces pensaba que me quería morir, que yo no quería estar más ahí, que yo no servía para nada, que no había estudiado, que ya era muy tarde, que eso ahora no; ahora no, yo quiero vivir, quiero... seguir siendo como era antes, pero con mejores pensamientos. (P3:23)

Estas mujeres con cáncer de mama no solamente luchan con su mejor actitud y fiereza, sino que sufren, les duele, sus heridas son profundas y los remedios a veces insuficientes:

Eso es indescriptible, claro eso es un algo como dijera yo... Algo horrible, algo que uno piensa ¿sí?, ¿me va a seguir avanzando, me va a seguir esto, cómo se detiene?, ¿cómo se podrá detener, qué me tomo?, ¿qué me bebo?, para así ayudarse uno mismo... Sí, eso es tremendo. (P1:66)

"Papi, me duele”, y él me decía: “¿pero qué le duele?", y yo le decía: “todo y nada". Yo no sabía qué me dolía. Yo decía: "si por lo menos me doliera una pata o me doliera algo"; pero no era una sensación tan horrible que yo decía: "pero yo tengo que salir delante de esto", y entonces y por la tarde bien. (P5:159)

\section{Discusión}

\section{Significado de la vida}

En este estudio se observó que el sentido de vida es significado, representado como el espíritu 
de lucha, la feminidad y la importancia de dejar una huella de género al demostrar que pese a la enfermedad no se deja de ser mujer, ni tampoco se descuidan sus funciones en los diferentes roles que desempeñan (esposa y madre principalmente). En contradicción con lo hallado por Giraldo-Mora y Arango (2008), las participantes de este estudio no hicieron referencia directa a los senos como símbolo de feminidad, pero sí a la pérdida como resultado del tratamiento oncológico. Es posible que esto se explique porque en este estudio se trata de mujeres enfermas, y en el de Giraldo-Mora y Arango (2008), de mujeres sanas. Se retomará esta discusión de la feminidad cuando se discuta el significado de la muerte.

Sin embargo, en lo que se refiere a la necesidad de trascender y al espíritu de lucha, las representaciones de las mujeres que participaron en este estudio son coincidentes con el planteamiento de Wong y Fry (1998), para quienes el sentido de vida es un constructo individual y un sistema cognitivo basado en el marco cultural de la persona que determina la toma de decisiones, actividades y metas, para dotar a la vida de sentido, propósito, valor personal y realización. De esta manera, también se ha argumentado que esta experiencia de encontrar el sentido permite a las personas trascender en experiencias intrusivas y negativas, promover una vida positiva y saludable. En el caso de este estudio es trascender la experiencia del cáncer pese a las dificultades que se presenten en el camino (Wong \& Fry, 1998). En consecuencia, con el planteamiento de Jaarsma et al. (2006), este hallazgo en particular permite ubicar esta estrategia de afrontamiento en la dimensión emocional del sentido de la vida, según la cual la lucha es característica de su construcción.

El sentido de la vida para las mujeres que participaron en este estudio también se basa en motivos intrínsecos y motivos extrínsecos. Para ellas, los motivos intrínsecos están relacionados con aquello que les permita seguir adelante y les evite caer o dejarse llevar por la enfermedad; en este caso sería lo que ellas representan como: espíritu de lucha, actitud de superación, optimismo y autoinstrucciones positivas. A partir de esto ellas se replantean su situación de vida, se fijan un nuevo proyecto de vida y buscan cumplirlo a cabalidad. Lo anterior es coherente con el planteamiento de Fryback y Reinert (1999), según el cual encontrar un sentido a la vida es importante cuando la persona enfrenta una enfermedad bastante seria, ya que la enfermedad causa en la persona cambios que pueden resultar siendo permanentes, lo cual obliga a la persona a reevaluar su sentido actual de la vida.

En cuanto a los motivos extrínsecos, están representados por los seres queridos que se preocupan por ellas y que las impulsan a seguir en su lucha en contra de la enfermedad, se convierten en un motor que las anima a levantarse luego de la caída y que, junto con metas que faltan por lograr, son una razón suficiente para superar la enfermedad. Lo anterior es coherente con el estudio longitudinal de Andreu et al. (2012), realizado con 102 mujeres con cáncer de mama no metastásico, en el cual encontraron que tanto el espíritu de lucha como el apoyo social tienen un papel protector en el afrontamiento del cáncer.

Como se ha venido mostrando, el significado de la vida cambia para estas mujeres con el diagnóstico de cáncer de mama. Eventos psicológicos y ambientales afectan este cambio. En específico, con respecto a los eventos ambientales, cabe retomar que, en Colombia, la oportunidad de citas médicas dentro del Sistema General de Seguridad Social en Salud dista de ser oportuna. Esto tiene como implicación directa la demora en el diagnóstico, el inicio del tratamiento o el seguimiento indicado como se ilustró en la figura 2. Lo anterior favorece el desarrollo de estrés y malestar emocional en los pacientes, pero en especial tiene implicaciones en la morbilidad y la mortalidad. Si bien la activación del cáncer varía según el curso natural de la enfermedad, su sospecha o diagnóstico requieren atención inmediata. Las mujeres con cáncer de mama lo saben y así lo expresaron en este estudio; pero 
su percepción de impotencia ante la dinámica del sistema de salud, el estrés de la espera, la angustia de ver o saber que el tumor crece, la exposición a la tramitología para autorizar exámenes y servicios médicos, entre otros, terminan por consolidarse en factores que median en los cambios o ajustes que se le dan al significado de la vida y de la muerte. Así, lo anterior se constituye en un elemento de enlace entre la vida y la muerte: a mayor probabilidad atención oportuna, menor probabilidad de empeorar o de morir. En contradicción con lo planteado en el Plan Decenal para el Control del Cáncer 2012-2021 (2012), pese a que estas mujeres son del régimen contributivo, ninguna tuvo diagnóstico en estadios iniciales; todas requirieron quimioterapia.

\section{Significado de la muerte}

El significado de la muerte está representado por la presencia del cáncer dentro del propio seno; es la muerte que yace dentro de ellas y que les recuerda su finitud. Este hallazgo es coherente con el planteamiento de Cicirelli (1998), según el cual, a partir del tipo de significado que la persona dé (positivo o negativo), a un evento como la muerte, las consecuencias para su vida serán similares a ese significado otorgado en cuanto a la forma de entender el mundo y la forma de enfrentarse a este, por ejemplo, a una enfermedad.

A su vez, la muerte está significada por los tratamientos oncológicos por ser generadores de un intenso sufrimiento y dolor; pero que aun así deben cumplirse para controlar la enfermedad y lograr en muchos casos la desaparición del cáncer. A lo largo de la investigación, también se encuentran posturas específicas asociadas al curso de la enfermedad, por ejemplo, el dolor, las náuseas, el vómito y el sufrimiento generados o asociados con la quimioterapia, hacen que eventualmente estas mujeres consideren la posibilidad de rendirse y de dejar de luchar por su vida.

$\mathrm{Al}$ analizar la representación que se hacen las mujeres sobre el significado de la muerte, es per- tinente mencionar que dentro de su discurso la palabra muerte al parecer ha sido evitada al máximo, a tal punto de desviar el tema hacia otras personas con cáncer (pero con otro tipo de cáncer diferente al propio). Cuando se les pregunta por la muerte, dicen de manera vehemente que si en algún momento llegaron a pensar en esa palabra, se dieron cuenta de que no era la opción más indicada para ellas y que era mejor no pensar en eso. Entonces, por esto el tema lo relacionan con otros enfermos y no con ellas, porque no contemplan esa posibilidad de morir y dejar sus planes sin ejecutar y mucho menos dejar a sus seres queridos. Al observar lo que refieren de la muerte, se encuentra que la cercanía que han tenido con esta les ha permitido resignificar muchas cosas y valorar otras tantas. Lo anterior es coherente con la propuesta de Marcu (2007), según la cual las experiencias personales de diferentes individuos con la muerte les han permitido renovar el valor de la vida; esto según King et al. (2009), quiere decir que luego de confrontar la finitud y la fragilidad natural de lo que es la vida, el valor o la apreciación de las personas cambia sustancialmente, a tal punto de darle mayor importancia a la vida.

Las representaciones que las mujeres del presente estudio han hecho de su condición de salud y que contribuyen a darle significado a la muerte son las siguientes en coherencia con el estudio de Salcedo (2008): el cáncer es persistente, el dolor que causa tiende a ser insoportable y su crecimiento rápido y desmesurado es asociado con la muerte y la putrefacción. A la vez, en el presente estudio no solo se encontró que las representaciones sobre la enfermedad subyacen al significado de la muerte; también subyacen a ella las representaciones de la cirugía oncológica y la quimioterapia. Las pacientes con cáncer de mama entienden que son alternativas de manejo/control de la enfermedad, que resultan efectivos en muchos de los casos, y en otros, llegar a acabar con el cáncer pero implican poner en riesgo su condición de mujer, generan sufrimiento y dolor y producen efectos secundarios desagradables. Lo anterior es coherente con 
el planteamiento de Anagnostopoulos y Spanea (2005), para quienes el cáncer de mama implica consecuencias negativas en el aspecto físico, la autoimagen y el atractivo de las mujeres, y así mismo con los resultados del estudio de Meretoja, Leidenius, Tasmuth, Sipilä y Kalso (2014), según los cuales el dolor tras los tratamientos oncológicos es persistente y es un problema clínico significativo que se ha mantenido a pesar de las estrategias terapéuticas. Por su parte, Bredal, Smeby, Ottesen, Warncke y Schlichting (2014) confirman en su estudio que, en efecto, las mujeres con cáncer de mama, independiente del estadio de la enfermedad, experimentan dolor y aquellas que han sido expuestas a tratamientos oncológicos combinados pueden desarrollar dolor crónico.

\section{Conclusiones}

Las mujeres con cáncer de mama experimentan cambios en su percepción sobre la vida y la muerte. El sentido de vida y de muerte, como se ha mostrado a lo largo de la investigación, tienen una alta relevancia en las construcciones de ideas asociadas a lo que significa tener cáncer; así, se construyen, se elaboran o se modifican, representaciones sociales que permiten resignificar una experiencia de vida ante la amenaza de la muerte. De esta manera, el significado de la vida va a girar en torno a la lucha que estas mujeres libran contra la enfermedad, así como la influencia de los motivos para vivir y la necesidad de que estos estén presentes a lo largo del proceso.

Por otro lado, la muerte, aun cuando se considera un proceso inherente a la condición humana, estas personas la ven como un asunto ajeno a ellas, pues el sentido que dan a la lucha constante contra la enfermedad las hace pensar (¿o desear?) que tal cosa no les podría ocurrir.

Este estudio también ayuda a entender el funcionamiento de las creencias comunes sobre la enfermedad, pues, como se evidenció en las entrevistas, para las mujeres con cáncer de mama es inconcebible que una persona no libre una ardua batalla en contra de la enfermedad; además, pensar en la posibilidad de abandonar el tratamiento supone una recriminación que proviene de los allegados del paciente.

Se espera que este tipo de investigaciones aporten a la comprensión de la experiencia de las mujeres con cáncer de mama que se encuentran en tratamiento con quimioterapia. Las representaciones que ellas tienen de su enfermedad, del tratamiento, del nuevo mundo que supone ser una mujer con cáncer pueden constituirse como elementos fundamentales para el abordaje psicológico e interdisciplinario de ellas, para comprender sus actitudes y sus necesidades emocionales y afectivas.

En conclusión, una mujer con cáncer de mama lucha por su vida y contra la muerte, enaltece su personalidad y no se deja mancillar por la mutilación de su seno enfermo ni por los temidos resultados de los químicos recibidos para "combatir" la enfermedad: el dolor, la alopecia y el vómito. Vencer el cáncer es a partir del momento de su identificación, el principal propósito de su vida al que posiblemente subyace que la probabilidad de morir aumenta el valor de la vida y el anhelo de vivir. El significado de la muerte está en el cáncer; el de la vida está en la lucha.

Unas intensas ganas de vivir y una silenciosa y temerosa resignación ante la muerte están presentes durante el curso de la enfermedad y del tratamiento en estas mujeres.

En lo que respecta a las limitaciones del presente estudio, se pone de relieve que los resultados no son generalizables, se limitan a las características del tipo de investigación y a las características sociodemográficas de la muestra. No obstante, esta es una primera aproximación al tema en población de mujeres con cáncer de mama, que da lugar a preguntarse por otros temas de interés que emergieron durante el proceso de análisis, como el significado del dolor, el temor a la muerte, el rechazo del suicidio en el proceso de enfermedad y aun en los momentos de mayor sufrimiento, la construcción 
de una imagen impecable y admirable y, por qué no, invencible. Esto supone que son necesarias investigaciones en las cuales se utilicen metodologías mixtas que permitan abordar otros grupos de mujeres con cáncer de mama, profundizar en la comprensión de las representaciones sociales de la vida y de la muerte para ellas; pero, a la vez, tener la posibilidad de sistematizar los resultados que se vayan obteniendo en instrumentos que se apliquen a muestras representativas de mujeres con este tipo de cáncer.

\section{Referencias}

Anagnostopoulos, F., \& Spanea, E. (2005). Assessing illness representations of breast cancer: A comparison of patients with healthy and benign controls. Journal of Psychosomatic Research, 58(4), 327-334. doi: 10.1016/j.jpsychores.2004.09.011

Anagnostopoulos, F., Slater, J., Fitzsimmons, D., \& Kolokotroni, P. (2011). Exploring global meaning in Greek breast cancer patients: Validation of the Life Attitude Profile - Revised (LAP-R). Psycho-Oncology (20), 419-427. doi: 10.1002/ pon. 1755

Andreu, Y., Galdón, M. J., Durá, E., Martínez, P., Pérez, S., \& Murgui, S. (2012). A longitudinal study of psychosocial distress in breast cancer: Prevalence and risk factors. Psychology \& Health, 27(1), 72-87.

Araya, S. (2002). Las representaciones sociales: ejes teóricos para su discusión. Cuaderno de Ciencias Sociales, 127, 1-82. Recuperado de http://unpan1.un.org/intradoc/groups/public/ documents/ICAP/UNPAN027076.pdf

Arman, M., \& Backman, M. (2007). A longitudinal study on women's experiences of life with breast cancer in anthroposophical (complementary) and conventional care. European Journal of Cancer Care (Engl), 16(5), 444-450.

Banchs, M. A. (2000). Aproximaciones procesuales y estructurales al estudio de las representaciones sociales. Papers on Social Representations,9, 3.1-3.5. Recuperado de: http://www.psr.jku.at/ PSR2000/9_3Banch.pdf

Beaver, K., Luker, K. A., Owens, R. G., Leinster, S. J., Degner, L. F., \& Sloan, J. A. (1996). Treatment decision making in women newly diagnosed with breast cancer. Cancer Nursing, 19(1), 8-19.

Bering, J. M. (2003). Towards a cognitive theory of existential meaning. New Ideas in Psychology, 21(2), 101-120. doi: http://dx.doi.org/10.1016/ S0732-118X(03)00014-X

Bonilla-Castro, E., \& Rodríguez-Sehk, P. (2000). La investigación en ciencias sociales: más allá del dilema de los métodos. Bogotá: Ediciones Uniandes-Norma.

Bower, J. E., Meyerowitz, B., Desmond, K., Bernaards, C., Rowland, J., \& Ganz, P. (2005). Perceptions of positive meaning and vulnerability following breast cancer: Predictors and outcomes among long-term breast cancer survivors. Annual of Behavioral Medicine, 29(3), 236-45. Recuperado de: http://link.springer. com.ez.urosario.edu.co/content/pdf/10.1207\%2Fs15324796abm2903_10.pdf

Bredal, I. S., Smeby, N. A., Ottesen, S., Warncke, T., $\&$ Schlichting, E. (2014). Chronic pain in breast cancer survivors: comparison of psychosocial, surgical, and medical characteristics between survivors with and without pain. Journal of Pain and Symptom Management, 48(5), 852862. doi:10.1016/j.jpainsymman.2013.12.239

Butkutè, R., \& Perminas, A. (2005). Giving meaning to life in illness for women with breast Cancer. Baltic Journal of Psychology, 6(2), 15-24.

Byock, I. (2002). The meaning and value of death. Journal of Palliative Medicine, 5(2), 279-288.

Castorina, J. A., Barreiro, A. \& Toscano, A. G. (2007). Dos versiones del sentido común: las teorías implícitas y las representaciones sociales. En J. A. Castorina (Coord.), Construcción conceptual y representaciones sociales: el conocimiento de la sociedad (pp. 205-238). Buenos Aires: Miño y Dávila. Recuperado de: http://www. 
pcyps.com/wp-content/uploads/2012/10/Castorina-Barreiro-Toscano-Dos_versiones_del_sentido_comun1.pdf

Cicirelli, V. G. (1998). Personal meanings of death in relation to fear of death. Death Studies, 22(8), 713-733.

Cicirelli, V. G. (2001). Personal meanings of death in older adults and young adults in relation to their fears of death. Death Studies, 25(8), 663-683.

Departamento Administrativo Nacional de Estadística. (s. f.). Estratificación socioeconómica: preguntas frecuentes. Bogotá. Recuperado de: http://www.dane.gov.co/files/geoestadistica/ Preguntas frecuentes estratificacion.pdf

Dupont, A., Bower, J. E., Stanton, A. L., \& Ganz, P.A. (2014). Cancer-related intrusive thoughts predict behavioral symptoms following breast cancer treatment. Health Psychology, 33(2), 155-163. doi: 10.1037/a0031131

Echebarría, A. (1991). Psicología social sociocognitiva: las representaciones sociales. Bilbao: Declée de Brouwer.

Fegg, M. J., Brandstätter, M., Kramer, M., Kögler, M., Haarmann-Doetkotte, S., \& Borasio, G. D. (2010). Meaning in life in palliative care patients. Journal of Pain and Symptom Management, 40(4), 502-509.

Fernandes, A., Cruz, A., Moreira, C., Santos, M. \& Silva, T. (2014) Social support provided to women undergoing breast cancer treatment: A study review. Advances in Breast Cancer Research, 3, 47-53. doi: 10.4236/abcr.2014.32007.

Fryback, P. B., \& Reinert, B. R. (1999). Spirituality and people with potentially fatal diagnoses. NursForum, 34(1),13-32.

Giraldo-Mora, C. (2009). Persistencia de las representaciones sociales del cáncer de mama. Revista de Salud Pública, 11(4), 514-525. Recuperado de: http://www.scielosp.org/pdf/rsap/v11n4/ v11n4a03.pdf

Giraldo-Mora, C. \& Arango Rojas, M. (2009). Representaciones sociales frente al autocuidado en la prevención del cáncer de mama. Investigación y Educación en Enfermería, 27(2), 191-200. Recuperado de: http://www.scielo.org.co/pdf/ iee/v27n2/v27n2a04.pdf

Gómez, M. (2007). la psicología de la salud en un hospital de cuarto nivel de complejidad. Psychologia. Avances de la Disciplina, 1(2), 159-179.

Ho, M. Y., Cheung, F. M., \& Cheung, S. F. (2010). The role of meaning in life and optimism in promoting well-being. Personality and Individual Differences, 48(5), 658-663.

Jaarsma, T., Pool, G., Ranchor, A., \& Sanderman, R. (2006). The concept and measurement of meaning in life in Dutch cancer patients. Psycho-Oncology, 16, 241-248. Recuperado de: http://share.eldoc.ub.rug.nl/FILES/root2/2007/ concanmeo/Jaarsma_2007_Psychooncology. pdf

Jim, H., \& Andersen, B. (2007). Meaning in life mediates the relationship between social and physical functioning and distress in cancer survivors. British Journal of Health Psychology, 12(Pt 3), 363-381.

Jodelet, D. (1989a). Folies et representations sociales. Paris: PUF.

Jodelet, D. (1989b). Represéntations sociales: un domain en expansion. En D. Jodelet (Dir.), Les représentations sociales. Paris: PUF.

Kahoe, R. D., \& Dunn, R. F. (1975). The fear of death and religious attitudes and behavior. Journal for the Scientific Study of Religion, 379-382.

Kállay, E. (2008). Investigation of the relationship between religious growth, positive effect, and meaning in life in a sample of female cancer patients. Cognition, Brain \& Behavior, XII(2), 161-182.

Kállay, E., \& Miclea, M. (2007). The role of meaning in life in adaptation to life threatening illness. Cognition, Brain \& Behavior, XI(1), 159-174.

King, L., Hicks, J., \& Abdelkhalik, J. (2009). Death, life, scarcity, and value: an alternative perspective on the meaning of death. Psycholsci, 20(12), 1459-1462. doi: 10.1111/j.14679280.2009.02466.x 
Livneh, H. (2000). Psychosocial adaptation to cancer: The role of coping strategies. Journal of Rehabilitation, 66(2), 40-49.

Marcu, O. (2007). Meaning making and coping: making sense of death. Cognition, Brain \& Behavior, 11(2), 397-416.

Meraviglia, M. (2006). Effects of spirituality in breast cancer survivors. Oncology Nursing Forum, 3(1), E1-E7. doi: 10.1188/06.ONF.E1-E7

Meretoja, T. J., Leidenius, M. H. K., Tasmuth, T., Sipilä, R., \& Kalso, E. (2014). Pain at 12 months after surgery for breast cancer. JAMA, 311(1), 90-92. doi:10.1001/jama.2013.278795

Mischel, W., \& Shoda, Y. (1995). A cognitive-affective system theory of personality: Reconceptualizing situations, dispositions, dynamics, and invariance in personality structure. Psychological Review, 102(2), 246-268. Recuperado de: http://faculty.washington.edu/mdj3/MGMT580/ Readings/Week\%203/Mischel.pdf

Moscovici, S. (1984). The phenomenon of social representation. En R. Farr, \& S. Moscovici, Social representations. Cambridge: Cambridge University Press.

Moscovici, S. (1998). The history and actuality of social representations. En U. Flick (Ed.), The psychology of the social (pp. 209-247). Cambridge: Cambridge University Press.

Murillo, R., Díaz, S., Sánchez, O., Perry, F., Piñeros, M., Poveda, C., et al. (2008). Pilot implementation of breast cancer early detection programs in Colombia. Breast Care, 3(1), 29-32. doi: 10.1159/000114446

Pardo, C., \& Cendales, R. (2010). Incidencia estimada y mortalidad por cáncer en Colombia 2002-2006. Bogotá: Instituto Nacional de Cancerología.

Parra, C., García, L., \& Insuasty, J. (2011). Experiencias de vida en mujeres con cáncer de mama en quimioterapia. Revista Colombiana de Psiquiatría, 40(1), 65-84.

Piñeros, M., Sánchez, R., Cendales, R., Perry, F., Ocampo, R., García, A.O., et al. (2008).
Características sociodemográficas, clínicas y de la atención de mujeres con cáncer de mama en Bogotá. Revista Colombiana de Cancerología, 12(4), 181-190.

Piñeros, M., Sánchez, R., Perry, F., García, O. A., Ocampo, R., y Cendales, R. (2011). Demoras en el diagnóstico y tratamiento de mujeres con cáncer de mama en Bogotá, Colombia. Salud Pública de México, 53(6), 478-485.

Ramfelt, E., Severinsson, E., \& Lutzen, K. (2002). Attempting to find meaning in illness to achieve emotional coherence. Cancer Nursing, 25(2), 141-149.

Ramos, S., Tamburrino, M. C., Aguilera, A., Capriatti, A., Lehner, P., \& Peña. (2013). Significaciones culturales, conocimientos y prácticas relativas al cáncer colorrectal, de mama $y$ de cuello de útero: un estudio sociocultural para orientar la política comunicacional de los programas de prevención. Buenos Aires: Instituto Nacional del Cáncer y Ministerio de Salud Presidencia de la Nación. Recuperado de: http://www.msal.gov.ar/images/stories/ bes/graficos/0000000224cnt-26-significaciones-culturales.pdf

Reker, G. T., Peacock, E.J., \& Wong, P.T. (1987). Meaning and purpose in life and wellbeing: A life-span perspective. Journal of Gerontology, 42(1), 44-49.

Rustoen, T., \& Begnum, S. (2000). Quality of life in women with breast cancer: a review of the literature and implications for nursing practice. Cancer Nursing, 23(6), 416-421.

Salcedo, H. (2008). Representaciones sociales y metáforas del cáncer en los siglos XVII y XVIII: una antología de lugares comunes. Antípoda, 6, 199-213.

Salmon, P., \& Valori, M. (1996). Measuring the meaning of life for patients with incurable cancer: The life evaluation questionnaire (LEQ). European Journal of Cancer, 32(5), 755-750.

Schnoll, R.A., Knowles, J.C., \& Harlow, L. (2002). Correlates of adjustment among 
cancer survivors. Journal of Psychosocial Oncology, 20(1), 37-59. Recuperado de: http://ac.els-cdn.com/0959804995006435/1s2.0-0959804995006435-main.pdf?_ti$\mathrm{d}=4 \mathrm{a} 76 \mathrm{e} 99 \mathrm{c}-\mathrm{c} 645-11 \mathrm{e} 2-\mathrm{bd} 6 \mathrm{c}-00000 \mathrm{aac}-$ b362\&acdnat $=1369601348 \_756348 f d b e d 5 b 2 b-$ 98c5582ae017c95cd

Sherman, A., Simonton, S., Latif, U., \& Bracy, L. (2010). Effects of global meaning and illness-specific meaning on health outcomes among breast cancer patients. Journal of Behavioral Medicine, 33(5), 364-377. Recuperado de: http://link.springer.com.ez.urosario.edu.co/ content/pdf/10.1007\%2Fs10865-010-9267-7. pdf

Sorajjakool, S., \& Seyle, B. (2005). Theological strategies, constructing meaning, and coping with breast cancer: A qualitative study. Pastoral Psychology, 54(2), 173-186.

Taylor, E. J. (1993). Factors associated with meaning in life among people with recurrent cancer. Oncology Nursing Forum, 20(9), 1399-1405.
Thompson, P. (2007). The relationship of fatigue and meaning in life in breast cancer Survivors. Oncology Nursing Forum, 34(3), 653-660.

Thorson, J., \& Powell, F. (1988). Elements of death anxiety and meanings of death. Journal of Clinical Psychology, 44(5), 691-703.

Uribe, A. G. (2012). Plan Decenal para el Control del Cáncer en Colombia, 2012-2021. s. d.

Wilde, D. J., \& Murray, C. D. (2009). The evolving self: Finding meaning in near-death experiences using Interpretative Phenomenological Analysis. Mental Health, Religion and Culture, 12(3), 223-239. doi: 10.1080/13674670802334910

Wong, P. \& Fry, P. (Ed.), (1998). The human quest for meaning: A handbook of psychological research and clinical applications. Mahwah, NJ, US: Lawrence Erlbaum Associates Publishers.

Zika, S., \& Chamberlain, K. (1992). On the relation between meaning in life and psychological well-being. British Journal of Psychology, 83(1), 133-415. doi: 10.1111/j.2044-8295.1992. tb02429.x
Fecha de recepción: 14 de julio de 2014 Fecha de aceptación: 17 de abril de 2015 